\title{
Protée
}

\section{L'idole au regard de la philosophie des images}

\section{Jean-Jacques Wunenburger}

Volume 29, numéro 3, 2001

Iconoclasmes : langue, arts, médias

URI : https://id.erudit.org/iderudit/030633ar

DOI : https://doi.org/10.7202/030633ar

Aller au sommaire du numéro

Éditeur(s)

Département des arts et lettres - Université du Québec à Chicoutimi

ISSN

0300-3523 (imprimé)

1708-2307 (numérique)

Découvrir la revue

Citer cet article

Wunenburger, J.-J. (2001). L'idole au regard de la philosophie des images. Protée, 29(3), 7-16. https://doi.org/10.7202/030633ar
Résumé de l'article

L'idole est une image paradoxale : image suprême, autosuffisante, elle inaugure aussi la mort de l'image qui ne vit que d'un rapport de dissemblance dans la ressemblance. Toute la philosophie de l'image en Occident, grecque (chez Platon), chrétienne (dans l'incarnation), moderne (dans l'esthétique du sublime), n'a peut-être fait que commenter ce paradoxe en reprenant sans cesse le patient travail de classification et de hiérarchisation des images afin de les sauver de leur contrefaçon idolâtre. 


\section{L'IDO LEAU REG ARD DE LA PHILO SO PHIE DES IM AGES}

JEAN -JACQUES W UNENBURGER

Toute image, mentale ou matérielle, est image de quelque chose et ne prend sens que par le jeu de ressemblance et dissemblance avec son référent. Creuser la différence dans l'image c'est risquer de la réduire à l'irréel, à la fiction, à la fantaisie, à l'insignifiant, mais surcharger à l'inverse la consistance de l'image c'est risquer de prendre la copie pour le modèle, de réifier la représentation, de télescoper le visible et l'invisible, le sensible et l'intelligible, le signifiant et le signifié, bref fabriquer une idole. L'idolâtrie constitue en ce sens une menace permanente de l'expérience spontanée, pré-réfléchie, des images matérielles, particulièrement attestée dans le registre religieux. La représentation imagée d'une divinité nous expose même à un paradoxe foncier, qui concentre à lui seul un grand nombre de problèmes de la représentation. En un sens, l'image religieuse constitue sans doute le prototype de l'image en général parce que l'image en général ne naît que de l'absence de ce qu'elle représente. Car l'imagination est bien production d'une représentation in absentia ${ }^{1}$. Tant que nous sommes en présence du réel, nous sommes des êtres percevants, pour lesquels l'imagination ne pourrait que devenir perturbatrice. Car toute image est image de quelque chose qu'elle remplace, parce que précisément elle ne se montre pas elle-même ${ }^{2}$. Or rien plus qu'un Dieu n'appelle l'image puisqu'il est en son principe même en retrait du visible et du sensible. Mais, inversement, nulle image n'exige davantage de croyance en sa propre consistance et réalité que l'image religieuse et l'image divine; d'abord parce que le sujet imaginant ne fait jamais l'expérience de son référent, ensuite parce que le référent en question renvoie lui-même le sujet au principe même de toute réalité, qui ne peut donc qu'irradier de sa vie propre son image ${ }^{3}$. L'image d'une divinité nous fait entrer dans le champ du sacré et induit un désir d'actualisation, de présentification, qui conduit à en faire une manifestation de l'invisible dans le visible. Ce paradoxe initial de l'image et de son référent entraîne que l'imagerie du divin risque précisément d'osciller entre une fiction pure, puisque le référent est inexistant pour l'expérience sensible, et un fétichisme, qui confond précisément en valeur l'être et son signe.

L'image a fait l'objet de descriptions, de classifications, de méthodologies, de normalisations esthétiques et même éthiques depuis l'antiquité grecque, dont 
l'héritage s'est mêlé à la foisonnante théologie de l'image propre au christianisme. Parallèlement, l'idolâtrie a été identifiée, spectrographiée, reconnue dans sa réalité complexe, dénoncée dans sa force insidieuse, à partir de positions métaphysiques, esthétiques, phénoménologiques, herméneutiques. Si plus d'une fois ce travail de pensée a conduit à l'extrême de l'iconoclasme, du fait d'une méfiance invétérée, il a permis aussi de sauver l'image du procès d'idolâtrie. Il s'agit donc ici moins de reprendre la question de la genèse de l'idolâtrie, ou de faire le bilan de ses méfaits, que d'établir combien la réflexion philosophique sur les images-idoles a précisément permis, tout au long de l'histoire, de discriminer entre mauvaise et bonne image, d'isoler l'image de son simulacre et même de libérer l'esprit de la séduction des images réifiantes et sidérantes sans pour autant priver la vie mentale de la pleine jouissance des images.

\section{LE JEU DE LA MIMESIS}

Historiquement, la question du statut ontologique de l'image s'enracine dans la tradition platonicienne grecque, qui l'a approché du point de vue de la génération de l'image, de la mise en image. L'image ne peut se comprendre que parce qu'elle a été faite à l'image d'un être qui tient lieu de modèle à reproduire. Il en a résulté une problématique canonique, dont bien des arguments perdurent, mais qui n'a pas toujours la simplicité et l'univocité qu'on lui prête. La pensée platonicienne a permis de distinguer les genres canoniques de l'icône et de l'idole, de rendre compte des méfaits de l'idolâtrie, tout en rendant possible une stratégie de libération et de désillusion, au profit de l'image homologique, apte à une fonction anagogique vers le vrai.

Certes pour Platon, le statut ontologique de l'image est généralement considéré comme négatif dans la mesure où l'image non seulement est seconde, inférieure à la réalité en soi et n'existe donc que par autre chose qu'elle-même, mais encore porte en elle la possibilité de contrefaire la reproduction apparemment ressemblante de la réalité en soi. En effet, pour Platon, l'image est définie comme ce qui vient mettre fin à la subsistance immuable d'une Forme essentielle (Eidos), qui est en soi et par soi; tout acte poïétique, d'un démiurge fabricateur du cosmos, ou d'un homme produisant un artefact technique ou artistique, consiste à arracher la Forme à sa singularité intelligible, connaissable par un pur acte d'intuition intellectuelle abstraite, pour la redupliquer dans une spatio-temporalité sensible. Créer de l'image (dans le mythos langagier ou dans les arts figuratifs), c'est projeter la Forme dans une configuration accessible aux sens. L'image résulte donc d'une pratique mimétique (mimesis) qui copie le modèle (paradeigma), le créateur ayant toujours les yeux fixés sur la forme génératrice. Ainsi le plan des images est toujours dérivé, subordonné, hétéronome, ce qui le rattache à un moindre-être qui doit toujours s'effacer au profit d'une intuition directe de l'être véritable. Ainsi, dans La République, Platon décrit l'action fabricatrice du menuisier comme une copie sensible d'un modèle (de lit, par exemple) unique et essentiel, le lit matériel n'étant qu'une image incomplète de la Forme en soi, "qui ressemble au lit réel mais sans l'être»4.

La dépendance ontologique de l'image se voit spécifiée et aggravée par la distinction de deux formes de mimesis: l'une, nommée eikastique, engendre des représentations sensibles qui sont homologues à leurs modèles et constituent dès lors des icônes (eikona): ainsi le menuisier fabrique-t-il un lit conformément au plan-type de ce que doit être la forme d'un lit; l'autre, nommée fantastique (Le Sophiste, p. 236c), fabrique d'autres images qui simulent la ressemblance apparente mais par une configuration physique dissemblante. Ainsi l'artiste sculpteur va déformer les proportions de sa statue pour qu'elles donnent l'illusion d'être vraies pour celui qui les voit de loin sur la place publique: ces artistes sacrifient donc «les proportions exactes pour y substituer, dans leurs figures, les proportions qui feront illusion" (Le Sophiste, p. 236a). Ainsi naissent des images fantomatiques, véritables idoles (eidola), qui se font prendre pour des copies isomorphes du paradigme mais n'en sont pourtant pas la reproduction exacte. 
Le but de la peinture n'est pas de même de

«représenter ce qui est tel qu'il est» mais «ce qui paraît tel qu'il paraît» (La République, p.598b). Dès lors l'art semble être complice d'une production de simulacres, dont la valeur négative vient non pas de ce qu'ils nous représentent des formes inexistantes, mais de ce qu'ils nous trompent en nous faisant croire à une ressemblance morphologique en trompe-l'œil. L'image fantasmatique (fantasma) perd de sa valeur ontologique parce que son imitation nous fait croire qu'elle est à l'image fidèle de l'être générateur. Comme le fait remarquer E. Fink, Platon a tendance à penser la génération mimétique de l'image d'après l'expérience spéculaire, dans laquelle le reflet symétrique de la Forme existe phénoménalement sans avoir d'être en soi.

Ce n'est pas par hasard que la critique platonicienne de la poésie se règle sur le modèle du miroir, sur l'image qui ne contient aucun élément ludique pour être copie au sens le plus fort. Et lorsqu'on fait du miroir une métaphore désillusionnante de l'art poétique, on affirme implicitement que le poète ne produit rien de réel, rien d'autonome; il ne fait que réfléchir comme un miroir, il reproduit sur le mode impuissant de la copie ce qui est déjà, en répétant simplement l'aspect superficiel de l'étant; le poète produit des apparences dans la sphère nulle des apparences. 5

Et de fait, dans La République, Platon ironise sur les pouvoirs magiques des créateurs en les comparant à des manipulateurs d'un miroir sur lequel ils font apparaittre, en le tournant sur lui-même, l'image du monde entier; mais alors, tel le peintre, ils ne créent que de l'apparence (phainomenen - p.596e) sans aucune réalité.

Peut-on pour autant conclure que toute théorie métaphysique de la préexistence d'un paradigme (essentialisme d'un monde intelligible et morphogenèse éidétique, dont la thématisation se retrouve à la Renaissance, dans le néoplatonisme anglais des $18^{\mathrm{e}}$ et $19^{\mathrm{e}}$ siècles, dans le romantisme européen, etc. ${ }^{5}$ ) condamne l'image à n'être que perte d'être? Si Platon semble en effet déceler dans le complexe regard-image une structure sidérante qui nous pousse à l'idolâtrie, il n'en reste pas moins qu'il n'y a là nulle fatalité. L'image, même confondue un instant avec le réel en soi, avec une figure isomorphe d'une Forme, garde une vie propre, une puissance d'animation de la pensée qui la rend capable de se déprendre du fétiche et de remonter à l'original. Car la puissance de tétanisation de l'image ne signifie pas une confusion irréversible entre l'être du référent et son paraître. Soit que l'on puisse découvrir l'insuffisance d'être de l'image, soit que paradoxalement on puisse prendre conscience que l'expression imagée contribue à une véritable augmentation d'être du modèle luimême. De ce point de vue il conviendrait en effet de mieux distinguer deux perspectives, que Platon tend parfois à superposer: d'un côté une approche de la création comme manifestation figurative de ce qui se tient caché dans un plan spirituel, qui permet de penser l'image comme expression et représentation limitée et déterminée de l'être. De l'autre côté, une approche ontologique des degrés d'être qui a pour but de fonder une théorie de la connaissance pure sans images sensibles, qui ne peut voir par conséquent en l'image qu'un détournement permanent de l'homme vers des apparences fallacieuses.

Or, chez Platon lui-même, la dissemblance objective de l'image, même dans le cas du récit mythique ou de la représentation plastique, n'est pas incompatible, dans l'expérience de la réceptivité, avec une conversion anagogique, qui lui permet de remonter vers la source essentielle de ce qui lui est représenté sans se trouver piégé par des simulacres. Autrement dit, la structure d'illusion de l'image, comme l'atteste l'allégorie de la caverne dans La République, laisse place à une recherche du modèle informant lui-même, puisque l'âme est potentiellement disposée vers la vérité qui sommeille en elle. Car l'âme reste toujours l'habitacle d'un typos (empreinte) de ce qu'elle met en image, inséparable d'un désir du modèle, qui la rend toujours apte à ressaisir l'original. À cet effet il suffit, comme le montre le texte de la libération du prisonnier, que l'âme soit stimulée par un tiers pour qu'elle se déprenne de la fascination des idoles. 
D'ailleurs, l'herméneutique contemporaine n'a pas manqué d'opérer une relecture de l'ontologie platonicienne tout en conservant le principe d'une filiation entre l'existence de l'image et une essence surplombante. Ainsi pour H.G. Gadamer, l'espace même de la mise en image, où ressemblance et dissemblance se mettent à jouer ensemble dans un rapport équivoque, constitue aussi une occasion pour l'image de participer à une manifestation de l'être et d'accéder par là même à un «surcroît d'être». En effet, l'image-tableau (Bild qui doit se distinguer de l'Abbild - copie) ne se ramène pas à l'image-miroir.

L'image-tableau a bien, dans le sens esthétique du mot, un être propre. Cet être qui est le sien propre en tant que représentation, qui fait précisément qu'elle est différente du modèle, lui donne, vis-à-vis de la simple copie, le caractère positif, celui d'être une image. Ce n'est pas un simple amoindrissement d'être, mais plutôt une réalité autonome. Que l'image ait une réalité propre, implique en retour que le modèle accède par l'image à la représentation. Il s'y représente lui-même - toute représentation de ce genre est un processus ontologique et s'incorpore au statut ontologique du représenté. Par la représentation le modèle acquiert pour ainsi dire un surcroît d'être. La teneur propre de l'image est ontologiquement définie comme une émanation $d u$ modèle. 7

Une telle réinterprétation implique une triple conséquence:

1) l'image devient un mode de manifestation de l'être qui appartient à l'essence de l'être:

On découvre que ce n'est que grâce à l'image que l'image-modèle (Urbild) devient proprement image-original (Ur-bild); autrement dit, ce n'est qu'en vertu de l'image que le représenté acquiert vraiment le caractère figurable (bildhaft); (ibid., p.69)

2) la mimesis n'est plus vraiment méconnaissance du modèle, mais «re-connaissance» dans et par le mouvement d'apparaitre de l'être dans l'image:

La relation mimétique implique donc non seulement que le représenté soit présent, mais que sa présence soit rendue plus authentique. L'imitation et la représentation ne sont pas seulement des répétitions et des copies; elles font connaître l'essence parce qu'elles ne sont pas simple «répétition»
(Wiederholung), mais «pétition» (Hervorholung) et qu'elles extraient de l'ouure ce qu'elle est réellement; (ibid., p.47)

3) c'est pourquoi enfin l'image, en tant qu'ostentive (zeigend), ne saurait être purement ressemblante au modèle: "Quiconque imite doit laisser tomber tels traits et souligner tels autres. Parce qu'il montre, il doit exagérer, qu'il le veuille ou non» (ibid., p.41).

Cette défiguration figurante de l'image a été d'ailleurs largement étudiée et validée par la peinture ou la littérature qui stylisent un portrait pour en assurer la ressemblance «vraie». Autrement dit, la dissemblance de l'image par rapport au représenté ne vient pas forcément ternir la présentation de la forme, mais au contraire participe, dans le champ du sensible, à son exposition, à sa mise en scène, comme l'avait remarqué, dans une autre perspective, plus réaliste qu'idéaliste, Descartes lorsqu'il soulignait ce mystère de la représentation artistique qui fait apparaître une chose à l'aide d'éléments figuratifs tout à fait hétérogènes:

[...] il faut au moins que nous remarquions qu'il n'y a aucunes images qui doivent en tout ressembler aux objets qu'elles représentent: car autrement il n'y aurait point de distinction entre l'objet et son image: mais qu'il suffit qu'elles leur ressemblent en peu de choses; et souvent même, que leur perfection dépend de ce qu'elles ne leur ressemblent pas tant qu'elles pourraient faire. Comme vous voyez que les tailles douces, n'étant faites que d'un peu d'encre posée çà et là sur le papier, nous représentent des forêts, des villes, des hommes, et même des batailles et des tempêtes, bien que, d'une infinité de diverses qualités qu'elles nous font concevoir en ces objets, il n'y en ait aucune que la figure seule dont elles aient proprement la ressemblance [...] en sorte que souvent, pour être plus parfaites en qualité d'images et représenter mieux un objet, elles doivent ne lui pas ressembler. 8

Ainsi tout en restant à l'intérieur d'un paradigme mimétique, on peut donner du jeu à la contrainte de ressemblance et donner une image du vrai qui n'est pas congruente à l'adéquation inhérente à l'intuition intellectuelle sans médiation. En tout cas, la réinterprétation herméneutique contemporaine ne 
s'éloigne du platonisme que pour mieux réactualiser l'approche de l'image propre au néoplatonisme antique et à certaines analyses gnostiques, pour qui l'être premier par excellence qu'est l'être divin ne peut sortir de la position immédiate de soi, où son être demeure encore indéterminé, en puissance, sans vie, qu'en se réfléchissant dans la figure déterminée d'une image. En produisant ainsi une image de soi en miroir, Dieu ne se redouble pas, inutilement ou fallacieusement, mais accède à la manifestation qui coïncide avec son propre passage à l'acte dans l'Être conscient de soi. Mais si l'image participe ainsi de l'épiphanie de Dieu, elle ne saurait mettre en question la transcendance même de son être qui ne peut jamais être épuisé par ses reflets.

\section{L'IMAGE COMME INCARNATION D'ÊTRE}

À côté de cette première tradition de philosophie des images, inséparable de la métaphysique grecque, la théologie chrétienne a développé une version de la mimesis sous forme d'incarnation et de transfiguration qui se distingue autant de la pensée grecque que de l'option iconoclaste juive. Dans la tradition biblique du judaïsme, en effet, l'humanité est faite à l'image de son créateur, mais en retour il est interdit à l'homme de fabriquer une représentation de la divinité sous peine de tomber dans l'idolâtrie, qui confond l'image et l'être et fait comme si la puissance illimitée et infigurable de Dieu était commensurable à une figure limitée. Le christianisme propose au contraire une autre forme de génération d'image: c'est Dieu en personne, déjà créateur d'images extérieures à lui, conformément au récit de la Genèse, qui devient luimême image, qui prend figure humaine. C'est pourquoi les premiers Pères de l'Église distinguent deux manières différentes de produire de l'image.

Ainsi, pour Origène:

Tantôt on appelle image ce qui est peint ou sculpté dans une matière comme le bois ou la pierre; tantôt on dit qu'un fils est

l'image de son père quand la ressemblance des traits du géniteur se retrouve sans erreur dans l'engendré. On peut, semble-til, appliquer le premier exemple à l'homme, fait à l'image de Dieu [...] À la seconde comparaison peut correspondre l'image qu'est le
Fils de Dieu dont nous parlons maintenant même en tant qu'il est invisible comme image du Dieu invisible [...] cette image implique l'unité de nature et de substance du Père et du Fils. En effet si tout ce que le Père fait, le Fils le fait pareillement, puisque le Fils fait tout comme le Père, l'image du Père est formée dans le Fils qui assurément est né de lui comme une volonté de lui procédant de l'Intelligence. [...] Notre Sauveur est donc l'image du Dieu invisible, le Père; en relation avec le Père il est vérité; en relation avec nous, à qui il révèle le Père, il est l'image par laquelle nous connaissons le Père que personne d'autre ne connaît si ce n'est le Fils et celui à qui le Fils aura voulu le révéler $[. . .]^{9}$

Autrement dit, le Christ, historiquement manifesté en ce monde, comme Fils de Dieu, est pensé comme une Image générée par Dieu le Père selon une relation de ressemblance interne et non de reproduction externe. La filiation remplace donc la reduplication, la généalogie remplace la création d'artefact. Ainsi par sa conception incarnationiste du Dieu, le christianisme se démarque bien, d'une part du judaïsme pour qui aucune image visible ne permet de remonter vers Dieu, et d'autre part du platonisme pour qui toute image demeure un artefact externe. Ainsi il existe au moins une Image, celle du Dieu visible en la personne de son Fils, qui est dotée d'une plénitude ontologique égale à celle de son principe et qui assure en même temps une fonction théophanique, de manifestation sans altération de l'Être absolu.

Cette promotion de l'Image sur le plan théologique ne va pas être sans conséquence dans l'histoire des représentations artistiques. La pratique des icônes, en particulier dans le christianisme byzantin, va même reposer sur un passage à la limite du principe métaphysique de la ressemblance par l'intermédiaire du mythe de l'icône achéiropoiétique. Dans les premiers siècles du christianisme, en effet, de nombreuses légendes vont identifier l'existence d'une image archétypique du Christ déposée miraculeusement sans main d'homme sur un support (Toile de Kamoulia en Cappadoce, Voile d'Édesse, jusqu'au Saint Suaire de Turin). Par ce dédoublement, par cette copie conforme, prélevée sur le modèle lui- 
même, la Face du Christ demeurerait visible pour les hommes après que par la mort il soit redevenu invisible. On assiste donc ici à une double génération de l'Image à partir d'un Principe informant: Dieu le Père infigurable, prototype de toute mise en Image, prend la Forme de son Fils visible, dont la forme visible archétypale se réinscrit dans une image sensible qui perdure après sa sortie du visible. Ainsi surgit une copie unique (bien que les légendes en aient multiplié les exemplaires) à partir de laquelle pourront être reproduites en nombre indéfini de nouvelles imagescopies. Ainsi, dans l'art de fabriquer des icônes de main d'hommes, se clôt une chaine ininterrompue d'images participant sans hiatus à l'Être même de Dieu, sans se confondre jamais avec lui.

Car sommes-nous en présence d'images réellement divines, Dieu est-il réellement présent, même partiellement, dans l'image au point que l'image pourrait tenir lieu de présentation en personne de Dieu? Tel était bien le fond du débat entre iconodoules et iconoclastes dans l'Église orientale ${ }^{10}$. Philosophiquement on peut sans conteste soutenir que si l'icône apparaît comme la consécration maximale d'une théophanie par l'Image, dans son fondement l'être de l'image ne peut être confondu avec l'Être auquel la divinité participe, et que, bien au contraire, l'ontophanie qu'elle permet repose précisément, et paradoxalement, sur le travail de l'incommensurabilité ou du vide dans la représentation, et ce pour plusieurs raisons. D'une part, l'icône se distingue théologiquement du sacrement de l'Eucharistie dans laquelle l'Église reconnaît une présence réelle du Christ, alors que l'image n'est qu'une re-présentation, la plus haute possible ${ }^{11}$; ensuite, l'icône est d'autant moins assimilable à une présence sensible de Dieu, malgré toutes les légitimations de filiation, que sa fonction n'est pas tant de servir à regarder Dieu que de faire l'expérience d'être regardé par lui. En effet, il s'agit moins pour le fidèle de contempler l'icône pour voir face à face le visage du Fils de Dieu, que de se laisser voir par lui, d'être placé sous un regard qui veille sur lui (d'où l'importance du regard du Christ
Pantocrator dans les églises byzantines) sans que nous puissions le voir autrement qu'avec nos yeux d'homme. L'icône nous rend attentif à la présence de Dieu sans que nous puissions le voir en lui-même, autrement qu'il ne nous apparaît. Cette asymétrie fait bien de l'icône une figure visible qui nous invite à passer au-delà du visible, à remonter vers l'Infigurable. C'est bien pourquoi d'ailleurs l'Église orthodoxe prescrit de ne jamais adorer les images comme si la divinité y prenait vraiment corps, mais tout au plus de les vénérer comme participant de la sainteté, comme expression finie de l'Infini ${ }^{12}$. C'est pourquoi le statut ontologique de l'Image iconique chrétienne peut être compris, avant tout, comme l'a développé dans un contexte occidental Nicolas de Cues ${ }^{13}$, comme expression. Il existe bien un rapport entre Dieu et son Image dans la mesure où Dieu est descendu dans une figure, certes finie mais contenant "en réduction" l'infini; mais toute Figure ne se laisse regarder que de manière finie, de sorte que nous ne pouvons voir l'Infini immanent dans l'Image. Enfin, dans le caslimite de l'Image achéiropoiétique qui pourrait nourrir la tentation de sacraliser une image isomorphe, on ne se trouve en présence ni d'une doublure qui répéterait la Forme christique, instaurant une confusion entre l'original et la copie, ni d'une copie-simulacre au sens platonicien, mais d'une simple empreinte vide. En effet, dans la théologie chrétienne de l'image, l'icône, comme le Christ luimême, est inséparable d'une kénose, d'un processus d'évidage de soi-même, dans lequel l'être en soi se retire de soi pour apparaître, et où le passage à la manifestation va de pair avec un retrait qui empêche l'enfermement dans une détermination physique particulière. Dans la perspective des Antirrhétiques d'un Nicéphore combattant, par exemple, l'iconoclasme, l'image iconique se justifie à partir de la kénose même du Christ; selon Marie-Josée Baudinet,

[...] l'image se distingue de la réalité en ceci que dans la réalité,

"la nature ne peut être traversée par le moindre vide», alors que dans l'image, il en va tout autrement. C'est donc que le vide, corrélat de l'image, concerne au plus près et l'Incarnation $d u$ Christ en tant qu'image, et son image en tant qu'image de 
l'image. La question de savoir si le Christ que l'on voit dans l'icône ou sur l'icône est ou non ressemblant à son modèle, serait totalement vaine et absurde si elle reposait sur une quelconque plénitude. La schesis, c'est-à-dire la relation d'image, définit l'imitation comme circulation des indices de la présence et de l'absence. L'icône n'est pas pleine de chair christique, en quoi elle se distingue radicalement de la consubstantialité eucharistique. L'icône est vide, comme le fut le ventre de la Vierge qui n'enferma jamais la divinité, qui forma sa visibilité sans la capturer, sans la cerner, sans porter atteinte à son illimitation [...] ce qui revient à dire de manière paradoxale que dans une icône à proprement parler il n'y a rien à voir, absolument rien, car cet espace n'est bâti que pour la circulation des regards, et il se creuse jusqu'au vide pour mieux faire écho à la voix. ${ }^{14}$

Ainsi le christianisme sanctifie bien l'Image en en faisant la forme du processus d'humanisation de Dieu qui doit permettre d'assurer à nouveau, après la Chute originelle, la Rédemption de l'humanité et sa redivinisation. Mais si Dieu s'incarne dans l'image, sa propre Image n'accède à son plein accomplissement qu'après sa mort au visible, c'est-à-dire dans l'image du Christ Transfiguré. Là le Christ se montre une nouvelle fois aux pèlerins d'Emmaüs ou sur le mont Thabor dans son corps de gloire, corps de lumière immatérielle, mais en même temps corps vide qui a déserté la chair périssable et sensible, pure Image d'une présence-absence ${ }^{15}$.

On peut donc comprendre combien l'hellénisme et le christianisme, chacun à sa manière, ont permis de mettre en place une systématique spéculative facilitant le triomphe de l'image et son utilisation artistique et religieuse. Dans leurs principes et exégèses de l'image, Platon et christianisme ont à la fois rendu compte de la genèse des idoles et rendu possible une correction du pouvoir de fascination des fantasmes et simulacres, surtout lorsqu'il s'agit de la divinité.

\section{LE PATHOS DE L'INFINI}

Qu'advient-il de cette question de l'être de l'image dans l'esthétique moderne, qui naît au XVIII siècle, et pour qui la question de l'image ne se pose plus tellement par rapport à une cause, à une source génératrice (être à l'image ou à la ressemblance de), mais par rapport à l'effet que l'image produit sur un sujet affecté par elle? En effet l'esthétique, qui privilégie l'état de réceptivité du sujet sur l'être de l'image, va renforcer la tendance iconoclaste puisque l'image n'assure plus de véritable présentation de son référent suprasensible. La problématique objective de la représentation imagée - vérité et fidélité de l'image - va faire progressivement place à celle, subjective, de la résonance de l'image sur le Moi, de l'émotion et des mouvements de pensée qu'elle génère en aval. Comment dès lors aborder dans ce nouveau contexte la question ontologique de l'être de l'image? Quelles sont les nouvelles catégories d'analyse et comment réorientent-elles la compréhension de la nature de l'image et du processus de l'idolâtrie?

D'une part, l'esthétique moderne renonce à comprendre le plan éidétique, la source originale des œuvres comme un ensemble fini et connaissable de Formes ou d'Idées génératrices, que la théologie chrétienne avait d'ailleurs déjà progressivement rendu opaque pour l'homme dénaturé par la chute originelle ${ }^{16}$. Les représentations imagées renvoient à un ordre invisible qui a perdu ses déterminations au profit d'une illimitation à la mesure de son caractère total et absolu. Autrement dit, l'Infini, après avoir longtemps été associé à l'inachèvement du sensible (ce qui est sans forme), sert à présent à définir positivement le cosmos ou Dieu. Dès lors l'homme, dont les facultés de connaissance sont limitées, ne dispose plus d'une intuition directe de ce qui est audelà de nos sens; un tel éclatement ou retrait de la notion de Forme paradigmatique a ainsi permis dès la Renaissance de concevoir les œuvres d'art comme des imitations de la seule Nature visible par l'homme. Par ailleurs, le sujet, dans l'acte de création ou dans l'attitude de réceptivité, ne se définit plus d'abord comme un sujet de connaissance dont le plaisir esthétique serait inséparable d'une re-connaissance de l'Invisible dans le visible, mais au contraire comme un sujet dont le Logos cède la place au seul pathos. L'image devient donc ce par quoi l'homme fini, raisonnable, 
peut se donner à sentir ce qui le dépasse, ou le surpasse, c'est-à-dire l'Infini, l'Absolu, Dieu. À défaut de voir à travers l'image l'au-delà du visible, nous expérimentons seulement son retentissement affectif en nous. L'art ainsi n'est plus essentiellement représentation à partir d'une intuition suprasensible, mais présentation dans la sensibilité de ce qui excède, par son incommensurabilité, par sa disproportion, notre pouvoir de représentation ${ }^{17}$.

Telle est la fonction accordée à l'esthétique du sublime dès la fin du XVIII e siècle ${ }^{18}$, qui va radicaliser pour l'image ce nouveau statut entre le fini et l'infini. On peut en effet poser, à côté d'une esthétique du beau, dont le plaisir est conditionné par la perception désintéressée de formes finies, définies par l'harmonie et la convenance, conformes à des normes raisonnables, et s'accordant avec l'homme, une esthétique du sublime qui vient de ce que le plaisir esthétique résulte de l'effroi éprouvé, en toute sécurité, devant des formes terrifiantes par leur grandeur mathématique (immensité) ou leur grandeur dynamique (violence des forces), par exemple devant le spectacle de la nature déchaînée (tempête, torrents dans les Alpes, etc., chers aux romantiques).

Dans ce contexte, le sublime, d'une part, renvoie à un sentiment éprouvé subjectivement devant ce qui nous suggère analogiquement l'Infini; il résulte d'une impression de toute-puissance extérieure qui me fait éprouver ma propre impuissance devant ce qui est d'une autre nature; ainsi pour E.Burke:

Tout ce qui est propre à exciter les idées de la douleur et $d u$ danger; c'est-à-dire, tout ce qui traite d'objets terribles, tout ce qui agit d'une manière analogue à la terreur, est une source de sublime; ou, si l'on veut, peut susciter la plus forte émotion que l'âme soit capable de sentir [...]. Lorsque le danger et la douleur pressent de trop près, ils ne peuvent donner aucun délice; ils sont simplement terribles: mais à certaines distances, et avec certaines modifications, ces affections peuvent devenir et deviennent réellement délicieuses. 19

D'autre part, le sublime renvoie à une image dans laquelle l'imagination produit une représentation maximale de ce qui se révèle directement irreprésentable pour l'homme. Comme le souligne Kant, le sublime «nous oblige à penser subjectivement la nature elle-même en sa totalité comme présentation (Darstellung) de quelque chose de suprasensible, sans pouvoir objectivement réaliser cette présentation $" 20$.

Dans cette perspective, l'image n'est plus une émanation ou une imitation d'une réalité idéale et idéelle, mais une simple apparence créée par l'homme pour se donner une présentation indirecte, symbolique, de ce dont nous éprouvons la présence autour de nous, au-dessus de nous, mais dont nous ne pouvons plus produire effectivement une présentification directe. L'image ne fait que symboliser l'Infini, ne peut que transposer la donation du Tout inaccessible dans une partie, de l'Absolu lui-même en autre chose que lui. La conception du sublime romantique enlève donc à l'image toute fonction ontophanique, toute consistance ontologique qui proviendrait d'une source transcendante. La question de l'idolâtrie se trouve ainsi vidée de son sens, puisque l'image ne saurait plus être commensurable à de l'être et encore moins, en son principe philosophique, se confondre avec un être référent.

\section{LE TRAVAIL DU NÉGATIF}

Quelles conséquences peut-on attendre de cette révolution épistémologique de l'image? Le repli de l'image au rang de simple apparence dans laquelle le monde suprasensible ne fait plus apparition, ne vientil pas valoriser la dimension de négativité et de vide de l'image? Le développement de méthodes nouvelles de description des images artistiques, qu'elles soient phénoménologiques ou herméneutiques, ne consacret-il pas la mise hors jeu de la notion d'idole?

On peut d'abord, dans le prolongement du kantisme, creuser l'écart entre représentation et représenté, non seulement en récusant pour l'imagination créatrice toute saisie d'un monde invisible suprasensible, qui ne serait qu'illusion délirante (Schwârmerei, selon l'expression de Kant), mais aussi en faisant de la production d'images un pur jeu entre sensibilité et raison, une libre création de semblances phénoménales, ne renvoyant plus à une 
Forme génératrice. L'image comporte certes un audehors, à partir duquel nous pouvons penser ce qui excède notre perception, mais ce dehors est toujours produit du dedans, à partir d'un sujet fantastique qui se donne une représentation asymptotique d'un point aveugle (focus imaginarius de Kant ${ }^{21}$ ). Dans cette perspective, s'est d'ailleurs développée une interprétation nihiliste de la création d'images, déjà amorcée par le mouvement de l'Athenæum, largement repris par des courants déconstructivistes aujourd'hui, et dont on peut dégager deux énoncés significatifs.

D'une part, l'image comme apparence symbolique de l'Absolu ne saurait se voir conférer de réalité, puisqu'elle existe comme pure phénoménalité. Ce qui apparaît dans l'image n'est qu'une semblance qui me fait penser à un Etre absent, hors présence.

G. Deleuze se propose d'ailleurs de renverser le platonisme en abolissant précisément le renvoi de l'image à l'original qui n'a de sens que dans une logique classique de la représentation; l'image simulacre de Platon doit être libérée de toute tutelle ontologique et déployée comme seule instance de l'apparence, dégagée de la question du vrai ou du faux:

Le simulacre n'est pas une copie dégradée, il recèle une puissance positive qui nie et l'original et la copie, et le modèle et la reproduction. Des deux séries divergentes au moins intériorisées dans le simulacre, aucune ne peut être assignée comme l'original, aucune comme la copie. Il ne suffit même pas d'invoquer un modèle de l'Autre, car aucun modèle ne résiste au vertige du simulacre [...] La simulation c'est le phantasme même, c'est-àdire l'effet de fonctionnement $d u$ simulacre en tant que machinerie, machine dionysiaque. 22

D'autre part l'image, parce qu'elle n'est en rien captation, accueil de l'être, ne peut que perpétuellement vaciller, se dérober, à la manière d'un pur phénomène (fantasma platonicien), faire place sans cesse dans un flux continu à une autre image, sans que jamais puisse être rejoint un point de jonction entre le paraître et l'être. L'image est répétition sans fin d'elle-même parce qu'en elle-même elle manque de substance, de consistance. Privée d'être, elle se présente comme mouvement incessant (tropisme, conatus, tendance vers); l'image perd donc bien toute valence ontophanique, s'évide, ne dispose que d'une forme-informe, toujours disparaissant, nourrissant en fin de compte un nouvel iconoclasme. Dès lors, la création c'est le mouvement même par lequel on s'arrache à toutes les images pour instaurer en soi-même un vide, un abîme, origine de toute expression, écriture ou représentation:

L'ouvre demande cela, que l'homme qui écrit se sacrifie pour

l'œuvre, devienne autre, devienne non pas un autre, mais plutôt personne, le lieu vide et animé où retentit l'appel de l'œuvre. Là la parole ne parle pas, elle est, en elle rien ne commence, rien ne se dit, mais elle est toujours à nouveau et toujours recommence. 23

Ainsi, pour M. Blanchot, créer n'est plus accueillir des images miroitantes de l'être, mais, en traversant le miroir des images, rejoindre l'être en soi, un être infini, infigurable, innommable, bref néant ${ }^{24}$.

On peut donc soutenir paradoxalement qu'en disqualifiant la relation ontologique de l'image entre la représentation et le référent transcendant, en rabattant l'image sur la seule immanence et la semblance, ces mouvements philosophiques ont déjoué le piège de l'idolâtrie. Si l'image n'a plus de prétention à manifester de l'être, elle ne peut offrir davantage qu'un ensemble d'apparences flottantes, qui déjouent toutes les identifications et tous les attachements fantasmatiques. L'ultime déréalisation de l'image n'est-elle pas accomplie dans la philosophie sartrienne qui non seulement porte un coup fatal au paradigme de l'image-tableau, mais ramène l'image à une pure intentionnalité néantisante, qui permet de se déprendre du réel en un geste de liberté? Pour Sartre, en effet, le sujet imageant, rencontré surtout à l'occasion de l'image mnésique (image souvenir de Pierre absent), se comprend avant tout comme une conscience néantisante: dans l'image, mon rapport au monde réel perçu, qui rencontre des objets représentés, est nié; l'image n'est qu'un néant d'être servant de corrélat noématique à la noèse, la visée par la conscience d'un objet: 
Dire «j'ai une image de Pierre» équivaut à dire, non seulement "je ne vois pas Pierre», mais encore «je ne vois rien du tout». L'objet intentionnel de la conscience imageante a ceci de particulier qu'il n'est pas là et qu'il est posé comme tel, ou encore qu'il n'existe pas et qu'il est posé comme inexistant, ou qu'il n'est pas du tout [...] [donc] «l'image donne son objet comme un néant d'être». 25

Ainsi avons-nous pu restituer une longue filiation de démarches philosophiques qui, tantôt à partir d'une ontologie réaliste, tantôt à partir d'une esthétique subjectiviste, isole, relativise, déjoue, voire évince ou même exclut le piège de l'image idolâtrique. Il existe donc bien sous des problématiques et rhétoriques disparates et parfois inattendues une philosophie de l'image marquée par le souci d'invalider et de marginaliser l'idole. Si ces courants qui traversent l'histoire des idées en Occident accompagnent de fait une récession de la question religieuse de l'idole, et même une indifférence croissante à son égard, on peut cependant craindre qu'en abandonnant la question du leurre idolâtrique, ils aient laissé le soin à d'autres de s'en emparer. À titre de symptôme philosophique et culturel, la naissance et le développement de la psychanalyse, son travail en profondeur sur le fantasme et les projections, ne s'expliquent-ils pas pour une part par la désertion de la philosophie et par la conscience d'une permanence et d'une urgence à traquer les idoles? En s'engageant ainsi dans cette voie, une grande partie de la philosophie moderne des images n'a-t-elle pas minimisé, voire négligé une des plus sourdes menaces contre la vérité et la liberté, contrastant ainsi avec le souci des Anciens qui avaient bien pris la mesure du danger que faisait planer l'image idolâtre sur la vie même de la pensée?

\section{NOTES}

1. Voir E. Kant, Leçons de métaphysique, Paris, Librairie générale française, coll. «Le Livre de poche", 1993.

2. Voir notre Philosophie des images, Paris, P.U.F., coll. "Thémis", 1997.

3. C'est pourquoi un dieu produit lui-même des images, en plus des réalités. Thème monothéiste par excellence que l'on retrouve aussi dans la pensée grecque. Voir Platon, Le Sophiste, Paris, Les Belles Lettres, 1963.

4. Platon, La République, Paris, Les Belles Lettres, 1950, p.597a.

5. E. Fink, Le Jeu comme symbole du monde, Paris, Éd. de Minuit, 1970, p. 107.

6. Voir E. Panovsky, Idea, Contribution à l'histoire du concept de l'ancienne théorie des arts, Paris, Gallimard, coll. "TEL», 1989; R. Klein,

La Forme et l'Intelligible, Paris, Gallimard, coll. "TEL», 1983.

7. H.G. Gadamer, Vérité et Méthode, Paris, Seuil, 1996, p. 160sq.

8. R. Descartes, La Dioptrique, Euvres I, Paris, Garnier, p. 685.

9. Origène, Traité des principes I, 2-6, Lyon, Sources chrétiennes, p.9596.

10. Voir C. von Schönborn, L'Icône du Christ, Fribourg, Éd. universitaires, 1976.

11. Voir les idées de Nicéphore au 9e siècle dans F. Boespflug et N. Lossky (sous la dir.), Nicée II, Paris, Cerf, 1987.

12. J. -L. Marion, L'Idole et la Distance, Paris, Grasset, coll. "Figures", 1977.

13. N. de Cues, Du tableau, ou de la vision en Dieu, Paris, Cerf, 1986.

14. M.-J. Baudinet, «Économie et idolâtrie dans la crise de l'iconoclasme byzantin ", dans Image et Signification, Paris, La documentation française, 1983, p. 187.

15. Voir notre analyse «Métamorphoses du regard et transfiguration des formes dans l'art contemporain ", dans Le Visible et l'Invisible (sous la dir. de J.-P. Sylvestre), Dijon, Éd. universitaires, 1994, p. 38sq.

16. Voir le thème de la vision en énigme de Dieu chez saint Paul ou du miroir brisé de la théologie médiévale dans R. Javelet, Image et Ressemblance au XII e siècle de saint Anselme à Alain de Lille, Paris, Letouzey et Ané, 1967.

17. À l'exception de ceux pour qui l'imagination demeure une faculté visionnaire comme les tenants de l'illuminisme (Hamann, Swedenborg, Balzac, etc.).

18. Telle qu'on la trouve théorisée chez E. Burke dans Recherche philosophique sur l'origine de nos idées du sublime et $d u$ beau (en 1757), Paris, J. Vrin, 1973; ou chez E. Kant dans la Critique de la faculté de juger (1790), Paris, J. Vrin, 1965.

19. E. Burke, Recherche philosophique sur l'origine de nos idées du sublime et du beau, p. 69-70.

20. E. Kant, Critique de la faculté de juger, §29, remarque générale. Voir aussi J. L. Nancy (sous la dir.), Du Sublime, Paris, Belin.

21. Voir E. Escoubas, Imago mundi, Topologie de l'art, Paris, Galilée, 1986.

22. G. Deleuze, Logique du sens, Paris, Éd. de Minuit, 1969, p. 302-303.

23. M. Blanchot, Le Livre à venir, Paris, Gallimard, coll. "Idées », 1986, p. 293-294.

24. Voir M. Bilen, Le Sujet de l'écriture, Paris, Greco, 1989.

25. J.-P. Sartre, L'Imaginaire, Paris, Gallimard, p. 25. 